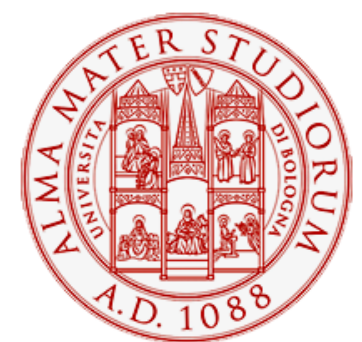

Alma Mater Studiorum - Università di Bologna DEPARTMENT OF ECONOMICS

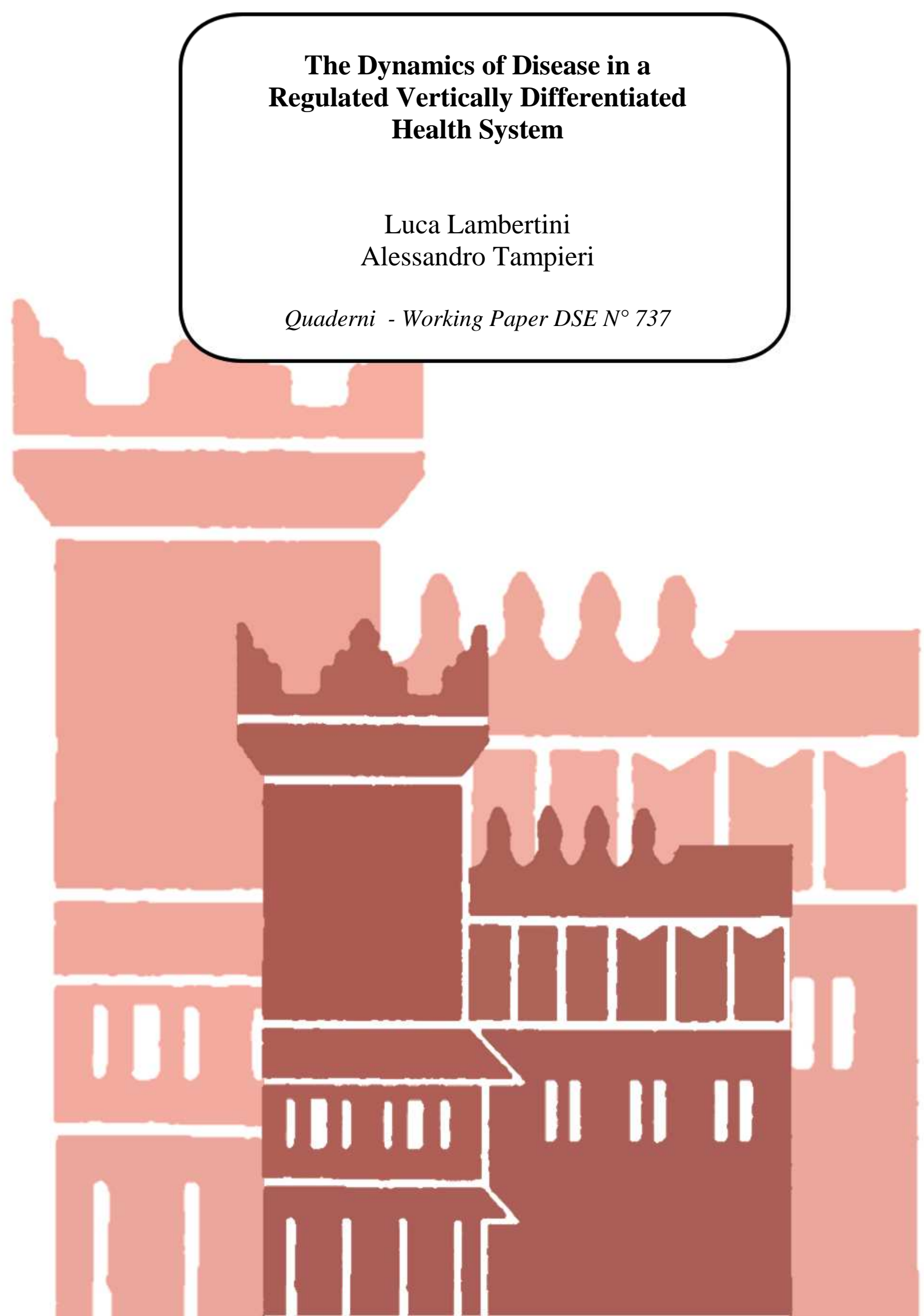




\title{
The Dynamics of Disease in a Regulated Vertically Differentiated Health System*
}

\author{
Luca Lambertini $^{\S}, \#$ and Alessandro Tampieri ${ }^{\S}$ \\ $\S$ Department of Economics, University of Bologna \\ Strada Maggiore 45, 40125 Bologna, Italy \\ luca.lambertini@unibo.it; alessandro.tampieri@unibo.it \\ \# ENCORE, University of Amsterdam \\ Roetersstraat 11, WB1018 Amsterdam, The Netherlands
}

March 3, 2011

\begin{abstract}
We build up a differential game to investigate the interplay between the quality of health care and the presence of an evolving disease in a duopoly where patients are heterogeneous along the income dimension. We prove unicity, stability and perfection of the open-loop Nash solution. Moreover, we identify the admissible parameter region wherein price regulation achieves the twofold objectives of ensuring cares to all patients and eradicating the disease.
\end{abstract}

JEL codes: C73, H42, I11, I18, L13

Keywords: health care markets, quality, dynamic games

*We thank Davide Dragone for precious comments and suggestions. The usual disclaimer applies. 


\section{Introduction}

So far, the theoretical literature on the quality of health care has extensively dwelled on the analysis of the provision of health care in the framework of multidimensional product differentiation combining vertical and horizontal dimensions (see Barros and Martinez-Giralt, 2002, Beitia, 2003, Brekke et al., 2006, Brekke et al., 2010, inter alia). However, a feature common to all the contributions belonging to this stream of research is that patients are supposed to be identical in their capacity of paying for medical cares, i.e., this approach generally leaves aside the typical assumption of hedonic tastes inherent to the analysis of quality choice in the theory of industrial organisation, dating back to Spence (1975) and Mussa and Rosen (1978). Additionally, the provision of health care is investigated in settings where the objective of health care, i.e., the disease, is only implicitly considered, so that these models could be interpreted as describing, in general, differentiated industries where firms are typically subject to price regulation.

To the best of our knowledge, Brekke et al. (2010) offer the first analysis of this issue in a dynamic game setup. Here, we set out with a twofold aim: to study the design of a health care regulation system where (i) the existence of a disease is explicitly accounted as a dynamic process, and (ii) patients differ in income and therefore also in their resulting willingness to pay for medical treatment.

We build up a dynamic duopoly with vertical differentiation where a high and a low-quality hospital set their health care levels and the market can be partially covered, in the sense that some individual may not receive health care. In the model, hospitals set their quality level non cooperatively at every instant. The demands for high and low quality differ depending on patients' income. Finally, we interpret price regulation in this model as a fine tuning device whereby the public agency adjusts prices so as to attain two goals: (a) ensure universal treatment, i.e., full market coverage, and (b) eradicate the disease.

The open-loop information structure is investigated first. Our results 
show that there is a unique steady state equilibrium where hospitals provide vertically differentiated services. We also single out the parametric condition required to ensure saddle point stability. Then, we show that there exists a unique vector of regulated prices ensuring indeed that every individual is cared for and the disease is completely eradicated. Finally, we model the feedback game to prove that the open-loop solution is in fact a degenerate feedback one, and therefore it is subgame perfect.

The remainder of the paper is structured as follows. The setup is laid out in section 2. Section 3 describes the open-loop Nash equilibrium. Price regulation policy is in section 4 . Section 5 briefly characterises the feedback game. Concluding remarks are in section 6 .

\section{The model}

We adopt a continuous time setup, where time is $t \in[0, T)$. We take a finite horizon as we are interested in finding out whether the disease can be eradicated in finite time. To investigate the optimal provision of health services, we rely on a variant of the vertical differentiation model with hedonic preferences originally due to Mussa and Rosen (1978), where individuals are indexed by a marginal willingness to pay for health care $\theta \in[\underline{\theta}, \bar{\theta}], \bar{\theta}>1$. $^{1}$ Over such interval, the population is uniformly distributed with unit density, so that its size is equal to one. Two hospitals serve the market, each one being characterised by a different quality level. The instantaneous measure of health care quality is $q_{i}(t), i \in\{L, H\}$, which is the control variable of hospital $i$. The price of quality $i$ at any time $t$ is constant at $p_{i}$, being regulated by the government or a public agency. For reasons that will become clear in the remainder, we allow for $p_{H}>p_{L}$.

By accessing the high-quality health service, an individual of type $\theta$ at-

\footnotetext{
${ }^{1}$ The marginal willingness to pay can be interpreted as the reciprocal of the marginal utility of income, whereby, if the latter is decreasing, $\theta$ increases in income. See, e.g., Tirole (1988, ch. 2).
} 
tains the following net instantaneous surplus: ${ }^{2}$

$$
U_{H}(t)=\theta+q_{H}(t)-p_{H}-D(t)
$$

where $D(t) \geq 0$ measures the level or intensity of disease suffered by this individual at time $t$. Instead, if the same individual resorts to the inferior quality care, the resulting net surplus is:

$$
U_{L}(t)=k \theta+q_{L}(t)-p_{L}-D(t)
$$

where $k \in(0,1)$ is a positive time-invariant parameter capturing the idea that gross satisfaction from receiving the low quality is lower. The third and last admissible case is that where an individual does not receive any health care; for the sake of simplicity, we set the corresponding utility level to zero which does not necessarily correspond to the death of the patient. To make sense of this normalisation, consider what follows. Parameter $\theta$ measures the individual taste for medical care in a hospital. If the patient is not being served, one may imagine that he/she receives some form of parental care at home, resulting in an alternative utility $n(t)-D(t)$, where $n(t)$ measures the instantaneous amount of the (unmodelled) parental care. It suffices to assume that $n(t)=D(t)$ at all times to economise on the number of endogenous variables.

In line of principle, we admit the possibility that the poorest section of the population be priced out of health cares. Accordingly, we set up the model under partial coverage. ${ }^{3}$ In order to construct the demand functions

\footnotetext{
${ }^{2}$ This specification of the utility function is borrowed from Colombo and Lambertini (2003).

${ }^{3}$ Although it would perhaps be natural to assume full coverage (as, e.g., in Brekke et al., 2010), we refrain from imposing it at the outset as it would cause the disease $D(t)$ to disappear from the profit functions of the two hospitals. This, in turn, would trivially imply that firms should be taxed in order to induce them to internalise this crucial aspect of their service.
} 
for the high- and low-quality services, we solve the indifference conditions:

$$
U_{H}(t)=U_{L}(t) \Leftrightarrow \widehat{\theta}(t)+q_{H}(t)-p_{H}-D(t)=k \widehat{\theta}(t)+q_{L}(t)-p_{L}-D(t),
$$

yielding

$$
\widehat{\theta}(t)=\frac{p_{H}-q_{H}(t)-p_{L}+q_{L}(t)}{1-k},
$$

and ${ }^{4}$

$$
U_{L}(t)=k \widetilde{\theta}(t)+q_{L}(t)-p_{L}-D(t)=0,
$$

yielding

$$
\widetilde{\theta}(t)=\frac{q_{L}(t)-p_{L}-D(t)}{1-k} .
$$

Hence, the demand functions are:

$$
x_{H}(t)=\bar{\theta}-\widehat{\theta}(t) ; x_{L}(t)=\widehat{\theta}(t)-\widetilde{\theta}(t) .
$$

On the supply side, we assume hospitals to be profit-seeking units. Prices are being regulated over the entire horizon of the game by a public agency, whose objectives will be discussed in detail in the remainder. Hence, hospital $i$ controls only the quality of its services $q_{i}(t)$ over time. The supply of health care entails the instantaneous cost $\Gamma_{i}(t)=c x_{i}^{2}(t)$, while any other costs are assumed away. Therefore, the hospital's instantaneous profit function is: ${ }^{5}$

$$
\pi_{i}(t)=\left[p_{i}-c x_{i}(t)\right] x_{i}(t) .
$$

${ }^{4}$ With parental cares $n(t) \neq D(t)$, the indifference condition (4) would instead write

$$
k \widetilde{\theta}(t)+q_{L}(t)-p_{L}-D(t)=n(t)-D(t) .
$$

\footnotetext{
${ }^{5}$ We disregard the possible presence of a lump-sum transfer to hospitals, as it is altogether immaterial in terms of the characterisation of equilibria and the viability of firms.
} 
The disease dynamics is represented by the following state equation:

$$
\dot{D}=s D(t)-v\left[q_{H}(t)+q_{L}(t)\right]
$$

where $D(t)$ is the intensity of the disease at time $t$ and the constant $s>0$ measures the rate at which the disease intensifies; parameter $v>0$ measures instead the effectiveness of health care.

The maximum problem of hospital $i$ can be formulated as follows:

$$
\max _{q_{i}(t)} \int_{0}^{T} \pi_{i}(t) e^{-\rho t} d t
$$

subject to the state equation (8) and the initial condition $D_{0}=D(0)>0$. The discount rate $\rho>0$ is assumed to be the same for both firms. The relative size of $\rho$ and $s$ will play a key role in shaping our results. To this regard, it seems natural to assume $\rho<s$ in order for hospitals to attach a proper importance to the future of patients, which additionally spills over positively to the present value of the profit flow. ${ }^{6}$

The game is fully non cooperative, with simultaneous play at every $t$, with two controls (quality levels, one for each player) and a single state (the intensity of the disease). Hence, the relevant solution concept is the Nash equilibrium, to be further qualified according to the nature of information attached to it. We consider first the open-loop information structure.

\section{The open-loop game}

Under open-loop information, firm $i$ has to choose its quality level $q_{i}(t)$ so as to maximise the Hamiltonian function:

$$
\mathcal{H}_{i}(t)=\left[p_{i}-c x_{i}(t)\right] x_{i}(t)+\lambda_{i}(t)\left[s D(t)-v\left(q_{H}(t)+q_{L}(t)\right)\right],
$$

\footnotetext{
${ }^{6}$ This specific point is also relevant in the field of environmental and resource economics, where low discounting increases the welfare of future generations (see, e.g., Stern, 2007; Nordhaus, 2007; and Weitzman, 2007).
} 
where $\lambda_{i}(t)$ is the costate variable associated with the state $D(t)$, under the initial condition $D(0)=D_{0}>0$, and the salvage value $\Omega_{i}(D(t))$ is set equal to zero, for reasons that will be clarified later. We are setting ourselves out to prove:

Proposition 1 The open-loop game yields a unique steady state equilibrium point, at

$$
\begin{gathered}
D^{O L}=\frac{\left[p_{H}(1+k)+2 p_{L} k-2 c\left(\bar{\theta}(1+k)-p_{H}-p_{L}\right)\right] v}{2(s-2 v) c} ; \\
q_{H}^{O L}=\frac{p_{H}(s-(1-k) v)+s p_{L} k-2 c\left[\left(\bar{\theta}-p_{H}\right) s+\left(p_{H}-p_{L}-\bar{\theta}(1-k)\right) v\right]}{2(s-2 v) c} ; \\
q_{L}^{O L}=\frac{2 c s p_{L}+s\left(p_{H}+p_{L}-2 \bar{\theta} c\right) k+\left[p_{H}(1-k)+2 c\left(p_{H}-p_{L}-\bar{\theta}(1-k)\right) v\right]}{2(s-2 v) c} .
\end{gathered}
$$

The condition $v(1-k)>s-\rho$ suffices to ensure that $\left\{D^{O L}, q_{H}^{O L}, q_{L}^{O L}\right\}$ be a saddle point equilibrium.

Proof. The necessary conditions are: ${ }^{7}$

$$
\begin{aligned}
& \frac{\partial \mathcal{H}_{H}}{\partial q_{H}}=\frac{2 c\left[p_{H}-p_{L}-q_{H}+q_{L}-\bar{\theta}(1-k)\right]+(1-k)\left[p_{H}-v(1-k) \lambda_{H}\right]}{(1-k)^{2}}=0 ; \\
& \frac{\partial \mathcal{H}_{L}}{\partial q_{L}}=\frac{2 c\left[D(1-k)+k\left(q_{H}-p_{H}\right)+p_{L}-q_{L}\right]+k(1-k)\left[p_{L}-k v(1-k) \lambda_{L}\right]}{k^{2}(1-k)^{2}}=0 ; \\
& -\frac{\partial \mathcal{H}_{H}}{\partial D}=\dot{\lambda}_{H}-\rho \lambda_{H} \Leftrightarrow \dot{\lambda}_{H}=\lambda_{H}(\rho-s) ; \\
& -\frac{\partial \mathcal{H}_{L}}{\partial D}=\dot{\lambda}_{L}-\rho \lambda_{L} \Leftrightarrow \\
& \dot{\lambda}_{L}=\frac{2 c\left[q_{L}-p_{L}-D(1-k)-k\left(q_{H}-p_{H}\right)\right]-k(1-k)\left[p_{L}-k(\rho-s) \lambda_{L}\right]}{k^{2}(1-k)^{2}} .
\end{aligned}
$$

\footnotetext{
${ }^{7}$ For the sake of brevity, henceforth we will omit the explicit indication of the time argument.
} 
The associated transversality conditions require $\lambda_{i}(T)=0$.

From (10-11), we may obtain both the dynamics of controls and the expressions of the optimal costates at any time. Solving (10-11) w.r.t. controls and differentiating the resulting expressions w.r.t. time, we obtain:

$$
\begin{gathered}
\dot{q}_{H}=\frac{2 c \dot{D}-(1+k)\left(\dot{\lambda}_{H}+k^{2} \dot{\lambda}_{L}\right) v}{2 c} ; \\
\dot{q}_{L}=\frac{2 c \dot{D}-k(1+k)\left(\dot{\lambda}_{H}+k \dot{\lambda}_{L}\right) v}{2 c} .
\end{gathered}
$$

Then, solving again (10-11) w.r.t. costates, we have:

$$
\begin{gathered}
\lambda_{H}^{*}=\frac{(1-k) p_{H}+2 c\left[p_{H}-p_{L}-q_{H}+q_{L}-\bar{\theta}(1-k)\right]}{(1-k)^{2} v} ; \\
\lambda_{L}^{*}=\frac{k(1-k) p_{L}+2 c\left[D(1-k)+k\left(q_{H}-p_{H}\right)+p_{L}-q_{L}\right]}{k^{2}(1-k)^{2} v} .
\end{gathered}
$$

Now, plugging (12), (13) and (15) into (14), we can rewrite the control equations as follows:

$$
\begin{gathered}
\dot{q}_{H}=\frac{1}{2 c}\left[(s-\rho)\left(p_{H}+k p_{L}\right)-k v(1-k) p_{L}-\right. \\
\left.2 c\left((s-\rho)\left(\bar{\theta}+q_{H}-p_{H}\right)+\left(k\left(q_{H}-p_{H}\right)+q_{H}+p_{L}\right) v+D(\rho-2 s+v(1-k))\right)\right] ; \\
\dot{q}_{L}=\frac{1}{2 c}\left[k\left((s-\rho)\left(p_{H}+p_{L}\right)-v(1-k) p_{L}\right)-\right. \\
\left.2 c\left((s-\rho)\left(k \bar{\theta}+q_{L}-p_{L}\right)+\left(k\left(q_{H}-p_{H}\right)+q_{H}+p_{L}\right) v+D(\rho-2 s+v(1-k))\right)\right] .
\end{gathered}
$$

Imposing stationarity on the dynamic system $\left\{\dot{D}, \dot{q}_{H}, \dot{q}_{L}\right\}$, we identify the coordinates of the unique pure-strategy open-loop Nash steady state equilibrium:

$$
D^{O L}=\frac{\left[p_{H}(1+k)+2 p_{L} k-2 c\left(\bar{\theta}(1+k)-p_{H}-p_{L}\right)\right] v}{2(s-2 v) c}
$$




$$
\begin{aligned}
q_{H}^{O L} & =\frac{p_{H}(s-(1-k) v)+s p_{L} k-2 c\left[\left(\bar{\theta}-p_{H}\right) s+\left(p_{H}-p_{L}-\bar{\theta}(1-k)\right) v\right]}{2(s-2 v) c} \\
q_{L}^{O L} & =\frac{2 c s p_{L}+s\left(p_{H}+p_{L}-2 \bar{\theta} c\right) k+\left[p_{H}(1-k)+2 c\left(p_{H}-p_{L}-\bar{\theta}(1-k)\right) v\right]}{2(s-2 v) c},
\end{aligned}
$$

where the superscript $O L$ stands for open-loop. Using (18-20), it is quickly checked that $\lambda_{H}^{*}=\lambda_{L}^{*}=0$, meeting thus the transversality conditions. Equilibrium outputs are $x_{i}^{O L}=p_{i} /(2 c)$.

In order to check stability, we have to evaluate the Jacobian matrix of the dynamic system:

$$
J=\left[\begin{array}{ccc}
\frac{\partial \dot{D}}{\partial D}=s & \frac{\partial \dot{D}}{\partial q_{H}}-v & \frac{\partial \dot{D}}{\partial q_{L}}-v \\
\frac{\partial \dot{q}_{H}}{\partial D}=2 s-\rho-v(1-k) & \frac{\partial \dot{q}_{H}}{\partial q_{H}}=\rho-s-v(1-k) & \frac{\partial \dot{q}_{H}}{\partial q_{L}}=0 \\
\frac{\partial \dot{q}_{L}}{\partial D}=2 s-\rho-v(1-k) & \frac{\partial \dot{q}_{L}}{\partial q_{H}}=-v(1+k) & \frac{\partial \dot{q}_{L}}{\partial q_{L}}=\rho-s
\end{array}\right],
$$

whose eigenvalues are:

$$
\xi_{1}=\rho-s ; \xi_{2}=s-2 v ; \xi_{3}=\rho-s+v(1-k)
$$

In order to ensure saddle point stability, the above eigenvalues must take different signs. A sufficient condition to this purpose is indeed $v(1-k)>$ $s-\rho$, whereby $\xi_{1}<0$ for the assumption that $\rho<s$, and $\xi_{3}>0$, irrespective of the sign of $\xi_{2}$. It is worth noting that the positivity of $\xi_{3}$ implies that the net growth rate of the disease must be more than offset by the marginal effectiveness of health cares evaluated by patients belonging to the lower section of the income distribution.

It is worth noting that at the steady state, for a given price vector, (i) full market coverage does not obtain in general, and (ii) the sub-population of patients being treated become chronic, i.e., as $D^{O L}$ is constant but positive. We'll come back to these issues in the next section. 
An obvious complement to Proposition 1 consists in assessing the effects of variations in prices on the equilibrium quality levels $q_{i}^{O L} .8$ The relevant partial derivatives are

$$
\begin{gathered}
\frac{\partial q_{H}^{O L}}{\partial p_{H}}=\frac{(1+2 c) s-(1+2 c-k) v}{2(s-2 v) c} \\
\frac{\partial q_{L}^{O L}}{\partial p_{L}}=\frac{(2 c+k) s-2 c v}{2(s-2 v) c}
\end{gathered}
$$

implying a non-monotonicity result that we may formulate in the following:

Corollary 2 For all $s>2 v, \partial q_{i}^{O L} / \partial p_{i}>0, i=H, L$. The same holds for all $s \in(0,2 c v /(2 c+k))$. Inside this range,

1. for all $s \in((1+2 c-k) v /(1+2 c), 2 v), \partial q_{i}^{O L} / \partial p_{i}<0, i=H, L$;

2. for all $s \in(2 c v /(2 c+k),(1+2 c-k) v /(1+2 c)), \partial q_{H}^{O L} / \partial p_{H}>0$ while $\partial q_{L}^{O L} / \partial p_{L}<0$.

That is, in quite extreme regions where the growth rate of the disease is either much higher or much lower than the marginal effectiveness of either medical care, each quality increases in its own price, all else equal. In the intermediate range, as $s$ decreases, first we observe a negative effect of price increases on quality levels, and then a further switch in the opposite direction. In particular, along $s=v, \partial q_{i}^{O L} / \partial p_{i}$ is negative for both firms.

Having characterised the optimal behaviour of the two firms and the reaction of optimal qualities to any price changes, we are now in a position to investigate the objectives of the public agency in charge of regulating prices, and the consequent design of the related measures.

${ }^{8}$ To this regard, there exists a lively debate in the literature, with contrasting results both on the theoretical side and on the empirical one (see, e.g., Gravelle and Masiero, 2000; Brekke et al., 2007; and Karlsson, 2007, for theoretical discussions, and Kessler and McClennan, 2000; Tay, 2003; and Gowrisankaran and Town, 2003, for empirical findings). 


\section{Regulating prices}

Given the nature of the problem at hand, we can argue that the regulator should manipulate prices so as to achieve a twofold objective, namely, to ensure that (i) the entire population has access to medical care (i.e., what we usually define as full market coverage), and (ii) the disease be completely eradicated, at least at the steady state equilibrium. We are about to show that there exists an admissible range for $\bar{\theta}$, wherein price regulation attains both objectives at the same time, as claimed in

Proposition 3 For all $\bar{\theta} \in\left(\frac{2 c(2 c+1-k)+(1-k) k}{2 c(1+k)+(1-k) k}, \frac{2 c+1+k}{1+k}\right)$, prices

$$
p_{H}^{R}=\frac{2 c[\bar{\theta}(1+k)-2(c+k)]}{1-k} ; p_{L}^{R}=\frac{2 c[2 c-(\bar{\theta}-1)(1+k)]}{1-k}
$$

ensure the universality of medical cares and the eradication of the disease in steady state.

Proof. This translates into solving the system

$$
\begin{gathered}
X^{O L}-1=0 ; \\
D^{O L}=0,
\end{gathered}
$$

w.r.t. $p_{H}$ and $p_{L}$. This yields the unique pair:

$$
\begin{aligned}
p_{H}^{R} & =\frac{2 c[\bar{\theta}(1+k)-2(c+k)]}{1-k} \\
p_{L}^{R} & =\frac{2 c[2 c-(\bar{\theta}-1)(1+k)]}{1-k},
\end{aligned}
$$

with

$$
\begin{gathered}
p_{H}^{R}>0 \forall \bar{\theta}>\frac{2(c+k)}{1+k} ; \\
p_{L}^{R}>0 \forall \bar{\theta}<\frac{2 c+1+k}{1+k} ; \\
p_{H}^{R}>p_{L}^{R} \forall \bar{\theta}>\frac{4 c+1+3 k}{2(1+k)},
\end{gathered}
$$


which also entail $x_{i}^{O L}>0$ and $x_{H}^{O L}>x_{L}^{O L}$. Moreover, it must also be true that $q_{H}^{O L}>q_{L}^{O L}$ in order to exclude the arising of a quality leapfrogging problem in correspondence of the regulated price vector. This requires:

$$
\bar{\theta}>\frac{2 c(2 c+1-k)+(1-k) k}{2 c(1+k)+(1-k) k} .
$$

Finally, it is easy to verify that

$$
\frac{2 c+1+k}{1+k}>\frac{2 c(2 c+1-k)+(1-k) k}{2 c(1+k)+(1-k) k}>\max \left\{1, \frac{4 c+1+3 k}{2(1+k)}, \frac{2(c+k)}{1+k}\right\} .
$$

\section{The degenerate feedback game}

It is well known that open-loop Nash equilibria are not, in general, subgame perfect (or strongly time consistent). Judging from the shape of the demand functions (6), the fact that $x_{H}$ is independent of $D$, while $x_{L}$ contains $D$ seemingly implies that the above open-loop solution indeed is not subgame perfect because the state variable appears in the system of first order conditions taken on controls. We are about to prove, instead, that the present game belongs to the class of so-called state-redundant or perfect games in which open-loop equilibria are degenerate feedback ones. ${ }^{9}$

The Bellman equation of hospital $i$ is:

$$
\rho V_{i}(D(t))=\max _{q_{i}(t)}\left\{\pi_{i}(t)+\frac{\partial V_{i}(D(t))}{\partial D(t)} \dot{D}\right\}
$$

where $V_{i}(D(t))$ is firm $i$ 's value function. We are going to prove the following:

Proposition 4 For any given price vector $\left\{p_{H}, p_{L}\right\}$, the game yields a unique

\footnotetext{
${ }^{9}$ See Mehlmann (1988, ch. 4) and Dockner et al. (2000, ch. 7), inter alia. Also note that, by imposing full coverage from the outset, the game would trivially become linear in the state variable, the latter appearing in the state equation only.
} 
feedback equilibrium coinciding with the open-loop one, whereby the latter is strongly time consistent.

Proof. The first order conditions are: ${ }^{10}$

$$
\frac{2 c\left[\bar{\theta}(1-k)-p_{H}+p_{L}+q_{H}-q_{L}\right]-(1-k)\left[p_{H}-v(1-k) \frac{\partial V_{H}(D)}{\partial D}\right]}{(1-k)^{2}}=0 ;
$$

$\frac{2 c\left[k\left(p_{H}-q_{H}\right)-D(1-k)-p_{L}+q_{L}\right]-k(1-k)\left[p_{L}-v k(1-k) \frac{\partial V_{L}(D)}{\partial D}\right]}{k^{2}(1-k)^{2}}=0$

from which we obtain optimal qualities

$$
q_{H}^{*}=\frac{p_{H}-2 c\left(\bar{\theta}-p_{H}-D\right)+k p_{L}-v(1-k)\left(\frac{\partial V_{H}(D)}{\partial D}+k^{2} \frac{\partial V_{L}(D)}{\partial D}\right)}{k\left[p_{H}+p_{L}-v(1-k)\left(\frac{\partial V_{H}(D)}{\partial D}+k \frac{\partial V_{L}(D)}{\partial D}\right)\right]-2 c\left(k \bar{\theta}-p_{L}-D\right)} ;
$$

Before proceeding any further, observe that plugging the above solutions into (6) one obtains the following expressions:

$$
\begin{gathered}
x_{H}^{*}=\frac{p_{H}-\frac{\partial V_{H}(D)}{\partial D}(1-k) v}{2 c} ; \\
x_{L}^{*}=\frac{p_{L}-\frac{\partial V_{L}(\stackrel{D}{)})}{\partial D} k(1-k) v}{2 c} .
\end{gathered}
$$

Since the low-quality output is the only possible source of a quadratic term in the entire problem, if $V_{L}(D)$ is linear the whole game is necessarily linear in $D$ as well, as there is no reason to suppose $V_{H}(D)$ to be quadratic. With this in mind, we may now turn to the explicit solution of the Bellman equations,

\footnotetext{
${ }^{10}$ Henceforth, we will omit the time argument for the sake of brevity.
} 
that simplify as follows:

$$
\begin{gathered}
p_{H}\left(2 \frac{\partial V_{H}(D)}{\partial D}(1+k+2 c) v-p_{H}\right)+ \\
\frac{\partial V_{H}(D)}{\partial D}\left[4 k p_{L}-(1-k)\left(\frac{\partial V_{H}(D)}{\partial D}(1+3 k)+4 \frac{\partial V_{L}(D)}{\partial D} k^{2}\right) v\right] v- \\
4\left[\frac{\partial V_{H}(D)}{\partial D}\left(s D+\left((1+k) \bar{\theta}-p_{L}-2 D\right) v\right)-\rho V_{H}(D)\right] c=0
\end{gathered}
$$

for firm $H$, and

$$
\begin{gathered}
p_{L}\left(4 \frac{\partial V_{H}(D)}{\partial D}(k+c) v-p_{L}\right)+ \\
\frac{\partial V_{L}(D)}{\partial D}\left[2 p_{H}(1+k)-(1-k)\left(2 \frac{\partial V_{H}(D)}{\partial D}(1+k)+\frac{\partial V_{L}(D)}{\partial D}(3+k) k^{2}\right) v\right] v- \\
4\left[\frac{\partial V_{L}(D)}{\partial D}\left(s D+\left((1+k) \bar{\theta}-p_{H}-2 D\right) v\right)-\rho V_{L}(D)\right] c=0
\end{gathered}
$$

for $L$. To reach a closed-form fully analytical solution, we have to conjecture the explicit form of the value function $V_{i}(D)$. On the basis of the above considerations, we pose that $V_{i}(D)$ is linear in $D$ :

$$
V_{i}(D)=\varepsilon_{i} D+\phi_{i}, i=H, L \text {. }
$$

Using the above functions, we may simplify the Bellman equations so as to obtain the following system:

$$
\begin{gathered}
4 D c(\rho-s+2 v) \varepsilon_{H}=0 \\
p_{H}\left[2 \varepsilon_{H}(1+k+2 c) v-p_{H}\right]+4 c \rho \phi_{H^{-}} \\
\varepsilon_{H}\left[4 c\left(\bar{\theta}(1+k)-p_{L}\right)-4 k p_{L}+v\left(\varepsilon_{H}(1+3 k)+4 \varepsilon_{L} k^{2}\right)(1-k)\right] v=0
\end{gathered}
$$

for hospital $H$, and

$$
4 D c(\rho-s+2 v) \varepsilon_{L}=0 ;
$$




$$
\begin{gathered}
p_{L}\left[4 \varepsilon_{L}(k+c) v-p_{L}\right]+4 c \rho \phi_{L}+\varepsilon_{L}\left[p_{H}(1+k)-\right. \\
\left.2 c\left(\bar{\theta}(1+k)-p_{H}\right)-v\left(2 \varepsilon_{H}(1+k)+\varepsilon_{L}(3+k) k^{2}\right)(1-k)\right] v=0,
\end{gathered}
$$

for $L$. Hence, it appears that our conjecture concerning the linearity of the value functions was indeed correct. ${ }^{11}$ The above equations are to be solved w.r.t. the unknown parameters appearing in the firms' value functions $\left\{\varepsilon_{H}, \varepsilon_{L}, \phi_{H}, \phi_{L}\right\}$.

From (36) and (38), one immediately obtains $\varepsilon_{H}=\varepsilon_{L}=0$, which means that the two firms' value functions are indeed constants, i.e., $V_{i}(D)=\phi_{i}$. Then, solving (37) and (39), we have:

$$
\phi_{H}=\frac{p_{H}^{2}}{4 c \rho} ; \phi_{L}=\frac{p_{L}^{2}}{4 c \rho} .
$$

Of course, the expressions appearing in (40) measure the discounted profit flows, as the feedback equilibrium profits accruing to firm $i$ are $\pi_{i}^{F}=\rho \phi_{i}$.

There remains to impose stationarity on the state dynamics, whereby $\dot{D}=0$ delivers:

$$
D^{F}=\frac{\left[p_{H}(1+k)+2 p_{L} k-2 c\left(\bar{\theta}(1+k)-p_{H}-p_{L}\right)\right] v}{2(s-2 v) c} .
$$

From (31), the equilibrium qualities are:

$$
\begin{gathered}
q_{H}^{F}=\frac{p_{H}(s-(1-k) v)+s p_{L} k-2 c\left[\left(\bar{\theta}-p_{H}\right) s+\left(p_{H}-p_{L}-\bar{\theta}(1-k)\right) v\right]}{2(s-2 v) c} ; \\
q_{L}^{F}=\frac{2 c s p_{L}+s\left(p_{H}+p_{L}-2 \bar{\theta} c\right) k+\left[p_{H}(1-k)+2 c\left(p_{H}-p_{L}-\bar{\theta}(1-k)\right) v\right]}{2(s-2 v) c},
\end{gathered}
$$

while from (6) we obtain the equilibrium output levels $x_{i}^{F}=p_{i} /(2 c), i=$ $H, L$, respectively. Hence, $X^{F}=x_{H}^{F}+x_{L}^{F}=\left(p_{H}+p_{L}\right) /(2 c)$.

This proves indeed that the open-loop equilibrium is subgame perfect,

\footnotetext{
${ }^{11}$ We have also checked the alternative possibility where the value function of $i$ is $V_{i}(D)=\gamma_{i} D^{2}+\varepsilon_{i} D+\phi_{i}$, finding that $\gamma_{i}=0$, so that the linear form obtains.
} 
and also implies that the regulatory measures outlined in the previous section trivially extend to the feedback game. And there is more to it. As prices appear in the firms' first order conditions, endogenising price regulation would require solving a Stackelberg game with the regulator in the leader's position. This would automatically imply the Stackelberg open-loop solution to be time inconsistent, as the interplay between prices and qualities involves that the game is controllable by the leader. ${ }^{12}$ One can tackle this problem by modelling a nondegenerate feedback Stackelberg solution where the leader's controls are taken to be state dependent in a linear way (see Dockner et al., 2000, pp. 134-135):

$$
p_{H}=a+b D ; p_{L}=w+z D,
$$

where $\{a, b, w, z\}$ is a vector of real numbers, and the regulator's problem consists in determining this vector for its own purposes. Given that the objective of the regulator is to achieve $D=0$ and $X=1$, (43) reduces to $p_{L}=a ; p_{H}=w$, where it suffices to set

$$
\begin{aligned}
& a=p_{H}^{R}=\frac{2 c[\bar{\theta}(1+k)-2(c+k)]}{1-k} ; \\
& w=p_{L}^{R}=\frac{2 c[2 c-(\bar{\theta}-1)(1+k)]}{1-k},
\end{aligned}
$$

to attain the same price regulation policy already characterised in the openloop game, the value of both $b$ and $z$ being altogether immaterial.

\section{Concluding remarks}

We have modelled the interplay between vertical differentiation and the endogenous evolution of a disease in a dynamic duopoly with heterogenous patients. In addition to the unicity, stability and subgame perfection of the

\footnotetext{
${ }^{12}$ For the concept of controllability in Stackelberg differential games, see Xie (1997) and Dockner et al. (2000, ch. 5).
} 
open-loop Nash equilibrium, our analysis yields a clearcut message as to the design of the price regulation policy, as there exists a parameter range wherein price regulation simultaneously delivers two eggs in one basket, namely, universal service and the eradication of disease. 


\section{References}

[1] Barros, P.P. and X. Martinez-Giralt (2002). Public and Private Provision of Health Care. Journal of Economics and Management Strategy 11: 109-133.

[2] Beitia, A. (2003). Hospital Quality Choice and Market Structure in a Regulated Duopoly. Journal of Health Economics 22: 1011-1036.

[3] Brekke, K.R., R. Nuscheler and O.R. Straume (2006). Quality and Location Choices under Price Regulation. Journal of Economics and Management Strategy 15: 207-227.

[4] Brekke, K.R., R. Nuscheler and O.R. Straume (2007). Gatekeeping in Health Care. Journal of Health Economics 26: 149-170.

[5] Brekke, K.R., R. Cellini, L. Siciliani and O.R. Straume (2010). Competition and Quality in Health Care Markets: A Differential-Game Approach. Journal of Health Economics 29: 508-523.

[6] Calem, P.S. and J.A. Rizzo (1995). Competition and Specialisation in the Hospital Industry: An Application of Hotelling's Location Model. Southern Economic Journal 61: 1182-1198.

[7] Colombo, L. and L. Lambertini (2003). Dynamic Advertising under Vertical Product Differentiation. Journal of Optimization Theory and Applications 119: 261-280.

[8] Dockner, E.J, S. Jørgensen, N.V. Long and G. Sorger (2000). Differential Games in Economics and Management Science. Cambridge, Cambridge University Press.

[9] Gowrisankaran, G. and R. Town (2003). Competition, Payers, and Hospital Quality. Health Services Research 38: 1403-1422. 
[10] Gravelle, H. and G. Masiero (2000). Quality Incentives in a Regulated Market with Imperfect Competition and Switching Costs: Capitation in General Practice. Journal of Health Economics 19: 1067-1088.

[11] Karlsson, M. (2007). Quality Incentives for GPs in a Regulated Market. Journal of Health Economics 26: 699-720.

[12] Kessler, D. and M. McClellan (2000). Is Hospital Competition Socially Wasteful? Quarterly Journal of Economics 115: 577-615.

[13] Mehlmann, A. (1988). Applied Differential Games. New York, Plenum Press.

[14] Mussa, M. and S. Rosen (1978). Monopoly and Product Quality. Journal of Economic Theory 18: 301-317.

[15] Nordhaus, W.D. (2007). A Review of the Stern Review on the Economics of Climate Change. Journal of Economic Literature 45: 686-702.

[16] Spence, A.M. (1975). Monopoly, Quality and Regulation. Bell Journal of Economics 6: 417-429.

[17] Stern, N. (2007). The Economics of Climate Change: The Stern Review. Cambridge, Cambridge University Press.

[18] Tay, A. (2003). Assessing Competition in Hospital Care Markets: The Importance of Accounting for Quality Differentiation. RAND Journal of Economics 34: 786-814.

[19] Tirole, J. (1988). The Theory of Industrial Organization. Cambridge, Cambridge University Press.

[20] Weitzman, M.L. (2007). A Review of the Stern Review on the Economics of Climate Change. Journal of Economic Literature 45: 703-724.

[21] Xie, D. (1997). On Time Consistency: A Technical Issue in Stackelberg Differential Games. Journal of Economic Theory 76: 412-430. 


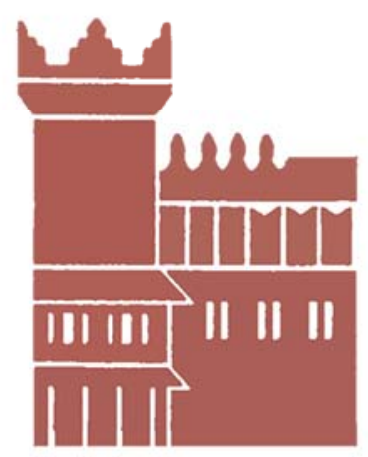

Alma Mater Studiorum - Università di Bologna DEPARTMENT OF ECONOMICS

Strada Maggiore 45

40125 Bologna - Italy

Tel. +39051 2092604

Fax +390512092664

http://www.dse.unibo.it 